\title{
EchoGéo
}

$8 \mid 2009$

Moyen-Orient : conflits et mobilités dans un espace mondialisé

\section{Tel-Aviv a cent ans ! 1909-2009: un siècle de globalisation au Proche-Orient}

\section{Caroline Rozenholc}

\section{OpenEdition}

\section{Journals}

Édition électronique

URL : https://journals.openedition.org/echogeo/10955

DOI : 10.4000/echogeo.10955

ISSN : 1963-1197

Éditeur

Pôle de recherche pour l'organisation et la diffusion de l'information géographique (CNRS UMR 8586)

Référence électronique

Caroline Rozenholc, "Tel-Aviv a cent ans ! 1909-2009 : un siècle de globalisation au Proche-Orient », EchoGéo [En ligne], 8 | 2009, mis en ligne le 09 mars 2009, consulté le 31 juillet 2021. URL : http:// journals.openedition.org/echogeo/10955; DOI : https://doi.org/10.4000/echogeo.10955

Ce document a été généré automatiquement le 31 juillet 2021.

EchoGéo est mis à disposition selon les termes de la licence Creative Commons Attribution - Pas d'Utilisation Commerciale - Pas de Modification 4.0 International (CC BY-NC-ND) 


\title{
Tel-Aviv a cent ans! 1909-2009: un siècle de globalisation au Proche- Orient
}

\author{
Caroline Rozenholc
}

\section{Tel-Aviv, globalisation et patrimoine}

1 Définitivement ancrée au Proche-Orient, «bulle» d'insouciance dans une réalité tourmenté, anti-Jérusalem archétypale et ouverte sur le monde ${ }^{1}$, Tel-Aviv surprend en permanence par son rythme et sa diversité. Espace de réception de la migration juive et, ces dernières années, de la main-d'œuvre "étrangère ", centre d'affaires, pôle culturel et de loisirs, marché immobilier en pleine effervescence, cette capitale économique fonctionne également, à certains égards, comme capitale administrative ${ }^{2}$. Tel-Aviv est aujourd'hui une ville mondiale ${ }^{3}$. La multiplication vertigineuse des gratteciels $^{4}$ aux enseignes internationales, comme la présence importante des travailleurs immigrés ${ }^{5}$ témoignent de ces mouvements de globalisation et matérialisent des dynamiques à l'œuvre, ici, comme dans l'ensemble de la région. Mais si la métropole donne à repenser l'inscription de réalités locales dans un monde globalisé - ou l'inscription locale de réalités globales - c'est aussi qu'elle est, simultanément, animée de dynamiques patrimoniales. Tel-Aviv ne vient-elle pas d'ailleurs d'être reconnue comme la «synthèse exceptionnellede l'urbanisme et de l'architecture moderne du $20^{\mathrm{e}}$ siècle », adaptés aux « conditions culturelles et climatiques » du lieu, et intégrés aux «traditions locales $»^{6}$ ? A l'aube du $21^{\mathrm{e}}$ siècle, Tel-Aviv s'est donc tournée vers son passé, pour revendiquer une profondeur historique que l'épaisseur temporelle de la région tend à lui dénier.

2 L'aspiration chère aux habitants de Tel-Aviv d'ancrer cette « création artificielle sans base réelle et sans future » (Vandervelde, 1930) ${ }^{7}$ a pourtant eu un écho majeur bien audelà des frontières nationales puisqu'en 2003, Tel-Aviv a été intronisée au panthéon du patrimoine mondial de l'humanité par l'Unesco. Un siècle aura donc suffi pour que 
cette ville «sans histoire» $(\text { Segev, 2001) })^{8}$ entre de plain-pied dans l'Histoire. De larges pans de la ville - bâtiments, rues et places - ont ainsi été réhabilités, laissant toutefois de côté des quartiers entiers de la ville. Cette mise à l'écart contribue à entériner un «narratif» dans lequel des espaces - le Sud de Tel-Aviv en général et en particulier les anciens quartiers juifs de Jaffa - pourtant significatifs de l'édification de Tel-Aviv peinent à s'inscrire ${ }^{9}$ (Rotbard, $2003: 32$ ).

Photo 1 - Tel-Aviv Nord et Sud (2008)

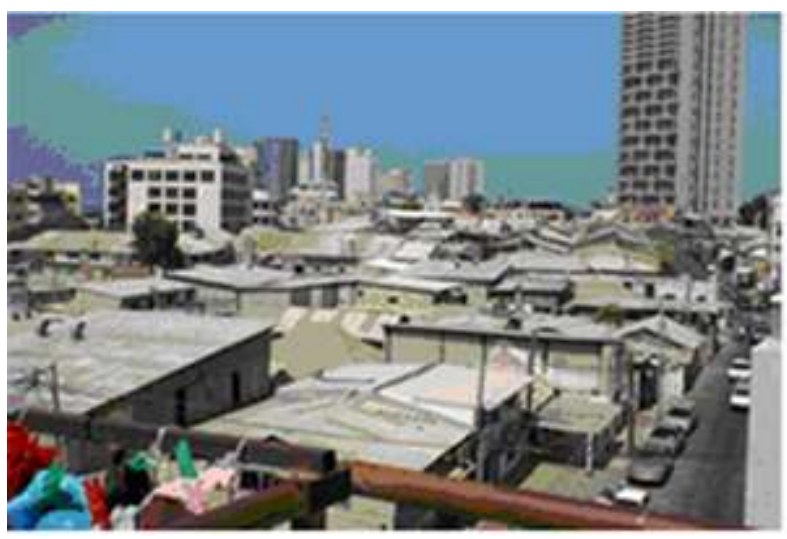

Au premier plan Tel-Aviv Sud : la partie industrielle du quartier de Florentin (Shrunat Wolowelsky) qui s'étend jusqu'à la rue de Jaffa. Au second plan (à droite), la tour Neve Tseddek rue de Jaffa. A l'arrière plan, les tours et buildings du centre ville.

3 L'histoire de la «Ville Blanche» ne fait donc pas l'unanimité (Weill-Rochant, 2003), même si pour la Municipalité elle se confond avec celle de Tel-Aviv : «l'histoire de la Ville Blanche, c'est histoire de Tel-Aviv $»^{10}$. Dans ce cas, comment un quartier de Jaffa en Palestine, créé en 1909 sur des fonds privés et soutenu par les organisations sionistes est-il devenu la «Ville Blanche", un «musée à ciel ouvert» des tendances du mouvement moderne en architecture et en urbanisme? Et comment Tel-Aviv, la "première ville hébraïque des temps modernes" en est venue à être identifiée au Bauhaus ; c'est-à-dire à devenir la vitrine d'une école allemande, d'un courant de pensée fondé à Weimar dans les années 1920 par l'architecte Walter Gropius (WeillRochant, 2006)?

\section{D'un quartier de Jaffa à la capitale célébrée du Style International}

4 La célébration de Tel-Aviv comme la «Ville Blanche » et de la «Ville Blanche » comme capitale du mouvement Bauhaus entremêlent plusieurs dimensions. Si en Israël, le terme «Bauhaus » désigne le style architectural d'inspiration Internationale (WeillRochant, 2006), nous retiendrons ici surtout la revendication d'une identité locale, matérialisée par un projet urbain moderniste et une architecture fonctionnaliste, l'un et l'autre importés en Palestine mandataire dans les années 1930. Partant de ce constat - de la mise en circulation des idées et de leur adaptation au contexte dans lequel elles s'implantent - cet article propose une « lecture » de la ville de Tel-Aviv comme ancrage successif d'idéologies et d'utopies européennes au Proche-Orient. Idéologies ancrées mais sauts d'échelles constants puisque dès ses débuts, Tel-Aviv semble s'être projetée 
hors d'elle-même. Tel-Aviv n'a-t-elle pas, par exemple, toujours été comparée à New York, la ville moderne (Levine, 2005)?

Photo 2 - Rue Kehilat New York (2008)

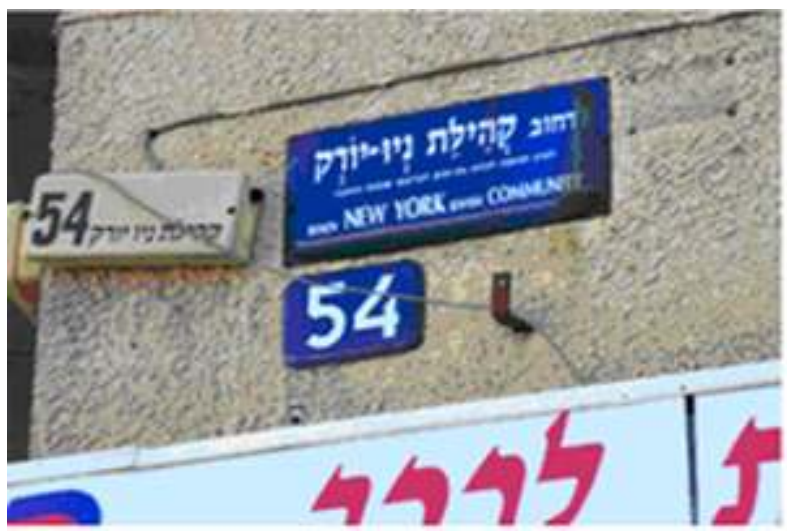

New York Jewish Community street, au sud de Tel-Aviv, dans le quartier de Ha'Tikva.

Modernisme donc, mais hygiénisme et fonctionnalisme auront également trouvé dans le cadre d'un développement national soutenu par une immigration massive ${ }^{11}$ les conditions de leur plein épanouissement. Et c'est dans cette continuité de la ville que l'époustouflant succès de l'architecture globale et l'intérêt grandissant des «starchitects » (Charney, 2007) pour Tel-Aviv doivent être réinscrits. Puisque Tel-Aviv, d'un quartier de la municipalité arabe de Jaffa à la ville globale des tours Yoo Tel-Aviv de Philippe Starck de respectivement 35 et 39 étages $^{12}$ se prête bien à une réflexion autour de l'articulation complexe d'échelles et de lieux, du local et du global, dans la durée.

Dans ce temps long - un siècle ! - trois moments marquants peuvent être distingués. Ensemble et successivement ils forment cette histoire urbaine aujourd'hui célébrée comme l'incarnation en un lieu de l'esprit d'une époque - le $20^{\mathrm{e}}$ siècle européen - alors même que le contexte géopolitique et socio-historique dans lequel elle s'est développée est si singulier. La première étape, la première rupture, s'opère avec ce qui date communément la naissance de la ville : la création en 1909 d'un nouveau quartier juif à Jaffa. Ce quartier juif de Jaffa n'est pas le premier à apparaitre au tournant du siècle dans la plaine côtière - cinq quartiers le précèdent de plusieurs années (Katz, 1986 : 405) dont le plus ancien, Neve Tsedek, de vingt ans ${ }^{13}$ - mais il se distingue des quartiers arabes et juifs existants en ce qu'il s'inspire du modèle des communautés allemandes chrétiennes, Whalhalla, Whilhelma et Sarona (Schlör, 1999; Weill-Rochant, 2006) présentent dans la région. Et le projet d'un quartier-ville planifié selon les règles de l'esthétique et de l'hygiène moderne « au lieu des conditions insalubres des logements de Jaffa » (Levine, 2005) est initié par une soixantaine d'habitants de Jaffa. 
Photo 3 - Maquette du quartier d'Ahuzat Bait (2008)

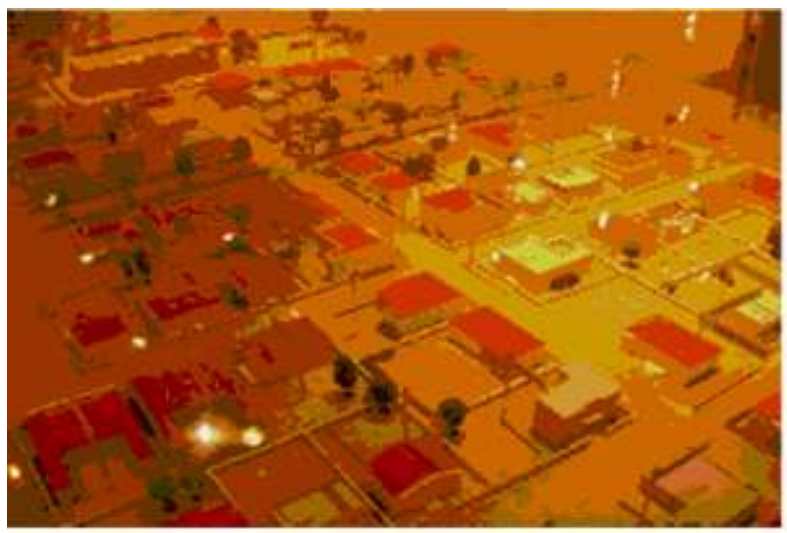

Le quartier d'Ahuzat Bait en tant que tel n'existe plus mais cette maquette exposée dans la tour Shalom (construite à l'emplacement du lycée Herzlyah) montre un parcellaire organisé autour d'un axe central, la rue Herzl, et de rues perpendiculaires le long desquelles se répartissent des maisons d'un, voire deux, étages. Le lycée au Nord (en haut à gauche) et la ligne de chemin de fer Jaffa-Jérusalem au Sud bornent le quartier.

7 L'entreprise, en obtenant le soutien financier de l'Organisation sioniste mondiale ${ }^{14}$, prend alors la dimension d'un projet national (Aleksandrowicz, 2008). Ce que le changement de nom du quartier, un an après sa création, d'Ahuzat Bait en Tel-Aviv soutient. En 1910, ce nouveau quartier juif est en effet rebaptisé du titre d'un roman de Théodore Herzl, le père du sionisme: Altneuland. Ancien nouveau pays, Tel-Aviv en hébreu ${ }^{15}$, publié en 1902 relate la création d'un Etat juif en Palestine qui, pour certains, se dessine déjà avec le développement de ce quartier-ville. L'instauration au sein de la municipalité arabe de Jaffa d'une idéologie sioniste-moderniste et son déploiement concret est un premier mouvement de mondialisation radicale qui concentre, au-delà des particularismes et des singularités de la situation, l'ensemble des dimensions de la modernité européenne ; nationalisme, socialisme, colonialisme (Wasserman, 2004).

Si d'aucuns voient de nouvelles perspectives s'ouvrir avec la matérialisation de ce projet, pour autant, Tel-Aviv n'est pas d'emblée destinée à devenir une grande agglomération. Le quartier est d'abord pensé comme une banlieue résidentielle dont tout commerce est proscrit ; une banlieue verte et spacieuse, branchée sur la ville-mère dont elle est issue : Jaffa. Tout bascule en 1921 avec des émeutes arabes qui vont très rapidement conduire à la séparation des entités juives et arabes et à l'octroi d'un statut autonome pour Tel-Aviv. Cette autonomie est entérinée en 1934 par la création d'une Municipalité à part entière. Et jusqu'en 1950, les deux municipalités vont fonctionner côte à côte. Dès la séparation de 1921, les commerçants juifs de Jaffa s'organisent et fondent Merkaz Mishari ${ }^{16}$, le premier centre d'affaires où seront dorénavant menées les activités commerciales, (relativement) indépendamment de Jaffa. Pour l'historien israélien Tom Segev, l'autonomie de Tel-Aviv - pas même la création d'une municipalité indépendante - est "l'accomplissement sioniste» le plus important depuis le début du Mandat britannique ; c'est une " pierre angulaire dans l'autonomie juive en Palestine » (Segev, 2001).

Deuxième moment: quelques années plus tard, l'administration britannique décide d'élaborer des «schémas directeurs» pour les grandes localités de Palestine. A TelAviv, cette impulsion gouvernementale rencontre la volonté du conseil communal « de rationaliser la planification de la ville » (Weill-Rochant, 2006) et de donner forme à une 
progression «aléatoire » qui jusqu'alors aura suivi les opportunités d'achat de terres. La mission de développer Tel-Aviv est confiée à l'urbaniste écossais Patrick Geddes. Et en 1925, Geddes propose un projet inspiré des villes-jardins. Il recommande de se défaire de l'architecture des banlieues européennes linéaire pour puiser, au contraire, dans la culture orientale (Metzger-Szmuk, 2004) et les nouvelles théories urbaines (Weill-Rochant, 2006). Le plan que Geddes a voulu entre « le pragmatisme et la vision, entre la pensée et l'action » (Metzger-Szmuk, 2004) est approuvé en 1927. Sa cité-jardin "suggère un nouveau rapport entre société et nature qui concilierait le social et la morphologie du paysage » (Metzger-Szmuk, 2004). Des détails de ce plan ${ }^{17}$ on retiendra surtout la multiplicité des espaces verts, même si tous n'ont pas été réalisés, mais aussi la création d'une hiérarchie de rues, entre grandes artères destinées au trafic, et rues résidentielles pour les piétons. Deuxième utopie et deuxième pensée globalisée, toutes deux ancrées dans les réalités locales de leur contexte. Le plan de Geddes a d'ailleurs largement été mis en œuvre et c'est à l'intérieur de son périmètre qu'est " contenue " la Ville Blanche. Il s'étend au Nord jusqu'à limite naturelle que constitue la rivière Yarkon et au Sud jusqu'aux quartiers déjà constitués au sein de la municipalité de Jaffa.

Troisième époque avec l'installation en Palestine de jeunes architectes qui fuient l'Europe en crise des années 30. L'arrivée de ces architectes, formés dans les écoles européennes, correspond à une grande vague d'immigration et un besoin urgent de logements. Entre 1929 et 1939, environ 180000 personnes arrivent en Palestine et près de 2700 bâtiments vont être construits entre 1931 et $1937^{18}$ pour répondre à cet afflux. Par ailleurs, les idées du style International et du fonctionnalisme du Bauhaus que ces architectes « importent » entre en résonance avec l'idéologie sioniste d'équité sociale et de revitalisation du peuple juif. Encore vingt ans, jusqu'en 1956, et se seront près de 4000 bâtiments de Style International construits à Tel-Aviv. La conjonction de ces différents facteurs explique bien comment Tel-Aviv en est venue à concentrer le plus grand nombre de bâtiments "Bauhaus» sur son périmètre. Et l'intense activité de construction va surtout s'inscrire à l'intérieur de la trame du plan Geddes, entre les rues Allenby, Begin, Ibn Gvirol, le Yarkon au Nord et la Méditerranée à l'Ouest (voir carte en fin d'article). Ce style introduit en Palestine par les architectes de l'école Bauhaus - Arieh Sharon, Yosef Neufeld et Ze'ev Rechter ${ }^{19}$, rejoints plus tard par Sam Barkai et Genia Averbuch ${ }^{20}$ et d'autres architectes venus d'Europe de l'Est - répond à la nécessité de construire rapidement des édifices fonctionnels.

\section{Conclusion}

11 Aujourd'hui, et depuis plusieurs années, Tel-Aviv a amorcé un nouvel élan. La ville se densifie et prend de la hauteur. Ainsi, des maisons d'un étage d'Ahuzat Bait, à la tour de verre et d'aluminium de "l'architecte du blanc», Richard Meir ${ }^{21}$, en passant par la reconnaissance de la ville comme inflexion « locale » d'une proposition architecturale internationale, Tel-Aviv se présente comme un laboratoire exceptionnel de réflexion sur l'articulation local/global. Pas tout à fait palimpseste, mais certainement pré-texte, le développement de la ville, si ramassé dans le temps, montre, à travers l'analyse de son projet urbain, comment se sont succédés les programmes idéologiques européens en Palestine, de fait, placée au cœur d'une certaine mondialisation. Aujourd'hui, la proposition Bauhaus pour Tel-Aviv semble à son tour répondre des mouvements de globalisation; avec la volonté d'affirmer l'identité historique d'une ville en transition 
rapide, une «ville du monde» (Kark et Y. Klein, 2008). Aujourd'hui comme hier, le passage des hommes et des idées ${ }^{22}$ façonne la ville, son tissu urbain comme la société qu'elle abrite et qui lui donne forme. Tel-Aviv, en ancrant localement des propositions importées, est devenue patrimoine mondial de l'humanité. Après un siècle de mondialisation, la ville continue donc de mêler les temporalités et les lieux.

Photo 4 - Au coin des rues Allenby et Rothschild (2008)

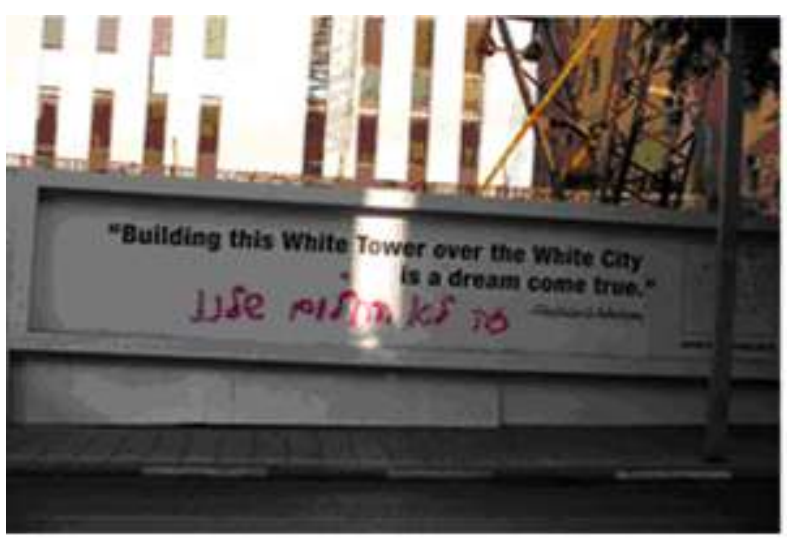

Site de construction de la tour Meir on Rothschild, Berggruen Holdings Company. « Building the White Tower over the White City is a dream come true, Richard Meier », panneau tagué en hébreu : «ze lo ha'khalom shelanou »: ce n'est pas notre rêve.

\section{Annexe1 - Carte de l'agglomération de Tel-Aviv Jaffa}

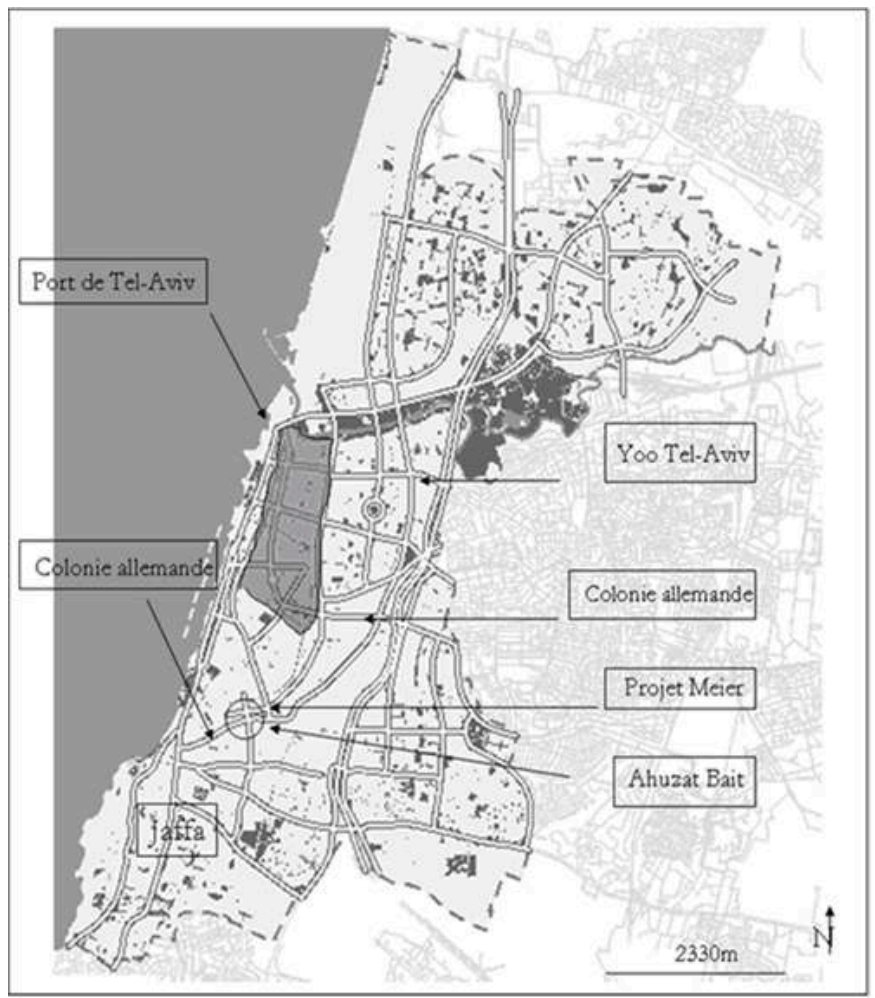

Repères géographiques (les colonies allemandes de Whalhalla et Sarona, Ahuzat Bait et le port de TelAviv, les projets de l'architecte Richard Meier et du designer Philippe Starck) au sein de l'agglomération de Tel-Aviv Jaffa. En grisé, le périmètre de la « Ville Blanche ». 


\section{BIBLIOGRAPHIE}

Aleksandrowicz, O, 2008. Kurkar, ciment, Arabes, Juifs : comment construit-on une ville hébraïque ?, Tsafon, $n^{\circ} 55$, p. 21-60

Alfasi, N, Fenster, T, 2005. A tale of two cities: Jerusalem and Tel Aviv in an age of globalization, Cities, vol. 22, $\mathrm{n}^{\circ} 5$, p. 351-363.

Bourel, D, Motzkin, G, 2002. Les voyages de l'intelligence : Passages des idées et des hommes, Europe, Palestine, Israël, Paris, Ed. du CNRS, coll. Cahiers du CRFJ, 335 p.

Charney, I, 2007. The politics of design : architecture, tall buildings and the skyline of central London, Area, vol. 39, n², p. 195-205.

Kark, R, Klein, Y, 2008. La démolition de la gymnasia Herzliya et la construction de la tour Shalom Meir. Début de l'américanisation de Tel-Aviv, Tsafon, $\mathrm{n}^{\circ}$ 55, p. 61-84.

Katz, Y, 1986. Ideology and urban development: Zionism and the origins of Tel-Aviv, 1906-1914, Journal of Historical Geography, n 12, vol. 4, p. 402-424.

Kipnis, B, 2004. Tel Aviv, Israel - A World City in Evolution: Urban Development at a Deadend of the Global Economy, Dela, n² 21, p. 183-193.

Levine, M, 2005. Overthrowing Geography. Jaffa, Tel Aviv, and the Struggle for Palestine, 1880-1948, Berkeley, University of California Press, $442 \mathrm{p}$.

Metzger-Szmuk, N, 2004. Des maisons sur le sable. Tel-aviv : mouvement moderne et esprit Bauhaus, Paris - Tel-Aviv, Ed. de l'éclat, 447 p.

Rotbard, S, 2003. White lies, white city, Territories, builders, warriors and other mythologies, Rotterdam, Witte de With, p. 26-40.

Schlör, J, 1999. Tel Aviv. From Dream to City, London, Reaktion Books, 309 p.

Segev, T, 2001. OnePalestine, Complete. Jews and Arabs under the British Mandate, New York, Owl Books, $612 \mathrm{p}$.

Wasserman, G, 2004. Editorial : Peut-on parler normalement d'Israël? Mouvements, $\mathrm{n}^{\circ}$ 33-34, p. 5-12.

Weill-Rochant, C, 2003. Mythes et constructions de Tel Aviv, Bulletin du Centre de recherche français de Jérusalem, $\mathrm{n}^{\circ} 12$, p. 80-96.

Weill-Rochant, C, 2006.Le plan de Patrick Geddes pour la "ville blanche » de Tel Aviv. Une part d'ombre et de lumière, thèse de doctorat, vol. 1, Université Paris 8 - Vincennes-Saint-Denis.

\section{NOTES}

1. «[F]or those Jews who dream of a 'Greater Israel', too, Tel Aviv is a suspect place, too open, too susceptible to contact with the outside world », Schlör $(1999: 210)$.

2. La plupart des ambassades étrangères ont leur siège à Tel-Aviv.

3. Les chercheurs israéliens analysent Tel-Aviv comme une ville globale depuis son entrée dans le marché international, l'accroissement des disparités sociales, la marchandisation des services sociaux et l'afflux de travailleurs immigrés, et en particulier des travailleurs illégaux (Levine, 2005 : 222). Voir aussi B. Kipnis (2004) N. Alfasi et T. Fenster (2005). 
4. On recense plus de 23 tours à Tel-Aviv, 179 à Haïfa, et 50 à Jérusalem, http:// www.emporis.com/en/.

5. Les travailleurs immigrés, venus de dizaines de pays d'Asie, d'Afrique, d'Amérique latine et d'Europe, se sont installés principalement dans les quartiers qui jouxtent la grande station de bus, au sud de la ville.

6. Site de l'Unesco, http://whc.unesco.org/fr/list/1096.

7. Le socialiste belge E. Vandervelde s'exprimant en 1930 sur Tel-Aviv, cité par Schlör (1999: 317).

8. Rapportant les propos d'un voyageur français qui dit de Tel-Aviv dans la années 1920 : TelAviv est une ville sans histoire et dépourvue de légendes.

9. Ces fractures au sein de la ville dessinent en réalité la permanence d'une frontière entre les Municipalités de Tel-Aviv et Jaffa jusqu'à « l'absorption » de l'une par l'autre.

10. http://www.white-city.co.il/english/index.htm.

11. L'immigration juive en Israël-Palestine s'est effectuée en vagues successives dont, la dernière, date des années 90 ; plus d'un million de ressortissants d'ex-URSS se sont alors installés dans le pays sur dix ans.

12. Yoo Tel-Aviv est le plus récent des projets de la société Yoo Ltd implantée dans une dizaine de pays.

13. Neve Tseddek est construit en 1887, Neve Shalom en 1890, Mahaneh Yehudah en 1903, Mahaneh Yosef en 1904 et Ohel Moshe en 1905.

14. L'association obtient un prêt du Keren kayemet le'Israel chargé de l'acquisition de terres en Palestine.

15. N. Sokolov qui traduit le roman de Herzl en hébreu choisit Tel-Aviv comme titre, « colline de ruines du printemps »; tel : une colline artificielle formée par l'accumulation de ruines et aviv : le printemps en faisant aussi référence à un passage biblique (Ezékiel, chapitre 3, verset 5).

16. Merkaz Mishari fait aujourd'hui partie intégrante du quartier de Florentin.

17. "The buildings will be constructed on plots of at least 560 square meters and each will be surrounded by a garden. The building area will be no larger than a third of the plot area. The distance between the buildings on each plot will be at least 3 meters and 4 meters from the street and 5 meters from the plot in the back of the building ", http://www.white-city.co.il/english/ index.htm.

18. http://www.white-city.co.il/english/index.htm.

19. Rechter est le premier à proposer des bâtiments sur pilotis, qui deviendront une des marques de la ville.

20. Genia Averbuch est architecte qui va dessiner la place Dizengoff.

21. R. Meier est le plus jeune récipiendaire du prix Pritzker en 1984. Il a, entre autres, réalisé le Musée d'Art contemporain de Barcelone et le Centre Getty de Los Angeles.

22. En référence au titre de l'ouvrage dirigé par Bourel et Motzkin (2002).

\section{RÉSUMÉS}

En mettant en perspective les grandes étapes du développement de Tel-Aviv, cet article propose de lire la capitale économique et culturelle d'Israël comme un lieu d'ancrage des idéologies du 20ème siècle européen au Proche-Orient. Sans faire l'impasse sur la mondialisation à l'œuvre 
dans la ville aujourd'hui, cette réflexion se déploie autour de la l'inscription récente de Tel-Aviv au patrimoine mondial de l'humanité. D'un quartier de Jaffa, Tel-Aviv est devenue la «Ville Blanche ", la ville du Bauhaus, la vitrine des tendances du mouvement moderne en architecture. L'enchaînement raisonné de ces différents moments permet de mettre à jour la manière dont cette ville « sans histoire » est entrée de plain-pied dans l'Histoire. Ainsi, des maisons d'un étage qui marquent la naissance de la ville, à la tour de verre et d'aluminium de «l'architecte du blanc ", Richard Meir, en passant par la reconnaissance de la ville comme inflexion "locale » d'une proposition architecturale internationale, Tel-Aviv se révèle comme un laboratoire exceptionnel de l'articulation local/global. Et l'engouement des architectes les plus prestigieux pour la ville ne fait que réactualiser Tel-Aviv comme lieu où jouent ces assemblages complexes d'espaces et de temporalités.

Putting Tel Aviv's development into perspective, this contribution suggests "reading" Israel's economical and cultural capital - Tel-Aviv - as the successful implementation of $20^{\text {th }}$ century European ideologies in the Middle East, Jaffa, Palestine. The starting point of this paper is the proclamation of Tel-Aviv as a World Cultural Heritage site for its unique urban and historical fabric, commonly known as the "White City" or the Bauhaus City. But how this city - generally considered as "devoid of history" - too set foot in History? And how from the one storey houses of the first neighborhood of Tel-Aviv, did it become within few decades the showcase of a renowned German architecture and arts school, the Bauhaus? How did it turn into the global city it is today within a century, with the most prestigious architects, the "starchitects", launching again Tel-Aviv as a complex mix of scales, places and moments. This chain of events, once analyzed and linked, shed light on Tel-Aviv as an amazing place for thinking the articulation of the local and the global.

\section{INDEX}

Mots-clés : Tel-Aviv, Moyen-Orient, globalisation, modernisme, architecture

Keywords : Tel-Aviv, Middle East, globalization, modernism, architecture

\section{AUTEUR}

\section{CAROLINE ROZENHOLC}

Caroline Rozenholc (caroline.rozenholc@etu.univ-poitiers.fr) est membre du Centre de recherche français de Jérusalem (Umifre 7 MAEE-CNRS) et de l'UMR 6588 (CNRS-Université de Poitiers) MIGRINTER 